\title{
Endoscopic Discectomy with Amniotic Allograft Tissue Implantation for Treatment of Lumbar Disc Herniation
}

\author{
Rudy Panganiban* \\ Doctor of Medicine, Fellow of the American Academy of Physical Medicine and Rehabilitation, USA
}

*Corresponding author: Rudy Panganiban, Doctor of Medicine, Fellow of the American Academy of Physical Medicine and Rehabilitation, USA, Tel: 5612451321; Fax: 5612451321; E-mail: rudoc@aol.com

Received date: September 19, 2017; Accepted date: September 27, 2017; Published date: October 03, 2017

Copyright: (c) 2017 Rudy P. This is an open-access article distributed under the terms of the Creative Commons Attribution License, which permits unrestricted use, distribution, and reproduction in any medium, provided the original author and source are credited.

\section{Introduction}

The clinical diagnosis of lumbar radiculopathy typically characterized by intervertebral disc encroachment within the neural foramen continues to constitute a significant portion of the patient population manifesting sciatica. Indeed, the progressive radiologic range spanning mild to severe disc bulge, disc protrusion, disc extrusion, and disc sequestration has rendered an array of treatment options aimed at restoration of function.

Within this study, patients manifesting radiating chronic low back pain extending primarily into the lower extremities corresponding to dermatomal extent and disc pathology conforming to disc protrusion/ extrusion radiographically was examined. Increasingly, minimallyinvasive treatment options have been sought and performed for pain reduction and restoration of function. Examples include nucleoplasty, percutaneous discectomy, laser disc decompression, and endoscopic microdiscectomy.

Treatment-wise in this study, all of the participants had prior received physical therapy, adjuvant modalities (moist heat/superficial cold/interferential electrical stimulation) without sustained benefit. Moreover, secondary to persistent pain and decline in function despite aforementioned conservative measures, patients, too, had prior received diagnostic and subsequent therapeutic transforaminal lumbar epidural steroid injections (40mg-80mg Kenalog or 8-10 mg Celestone) without sustained benefit as it relates to levels of pain and corresponding functional activities of daily living.

Medicinally, the test participants where prior progressed from NSAIDS to adjuvant analgesics including low-dose opioids (hydrocodone/oxycodone less than three tablets a day) without sustained benefit in regards to their pain nor function as well.

\section{Results}

In keeping with a minimally-invasive treatment model, endoscopic lumbar microdiscectomy was performed in the treatment population. A total of fifty two patients were randomized to receiving or not receiving allograft tissue implantation as part of the surgical procedure. $21 / 52$ patients received allograft tissue implantation intradiscally after endoscopic lumbar micro discectomy. VAS pain scale scores and SF-36 parameters (Role-Physical, Bodily-Pain) were measured at 2 weeks, one month, and six months. Physical therapy measures including postural retraining, biofeedback measures, core strengthening, dynamic lumbar stabilization exercises were implemented between weeks 2 and 4 .

$46 / 52$ of the total test population reported Visual analog scale reduction of at least 30 percent and improved function (SF-36) at week two. 44/52 reported sustained reduction of pain (at least 30 percent reduction) and functional improvement at one month.

At six months 40/52 test participants reported sustained parameter improvement.

In the positive test responders with regards to visual analog scale and SF-36 functional parameters 19 of the total of 21 patients receiving allograft tissue implantation were included. Consistently, at one month and at six months, the 19 allograft tissue recipients retained their functional benefit. Maintenance of SF-36 (Role-Physical, Bodily Pain) scores at the six month interval was statistically significant ( $\mathrm{p}$ less than 0.05 ) when compared to the positive responders not receiving allograft tissue implantation. Of the 22 positive test responders who did not receive allograft tissue implantation, 16 reported benefit at one month while 12 reported sustained functional benefit at six months ( $\mathrm{p}$ less than 0.5 ).

\section{Materials/Methods}

Radicular symptoms were defined as radicular pain with concomitant sensory and/or motor deficits involving the lower extremities. The exclusion criteria included axial pain (low back pain) only, history of prior surgery (laminectomy/fusion), history of spinal infection or inflammatory spine disease, history of vertebral compression fracture, and/or history of vascular claudication due to atherosclerotic disease involving the lower extremities. Axial and Sagittal T-1,T-2 weighted images from a 1.5 Tesla or better MRI scanner was utilized. According to the lumbar disc classification as proposed by Fardon et al, disc protrusion represents a focal displacement of disc material extending less than 25 percent of the circumference of the disc when viewed in the axial plane. This is contrasted to a disc extrusion where the edge of the herniated disc material at any point is greater than the distance between the edges of the base of the disc material. Patients who demonstrated radicular symptomology with disc protrusion anterior-posterior diameters measuring less than $6 \mathrm{~mm}$ with an intact posterior longitudinal ligament were included in this study. Classically, disc protrusions measuring greater than $6 \mathrm{~mm}$ have been addressed with open discectomy.

The technological advancement and evolution for anterior endoscopic and concomitant neural decompression (Rothstein) has allowed for more direct visualization and treatment of anterior disc protrusions and annular tears.

Utilizing a Ventral-Medial approach accessing, via the transforaminal approach, the "safe" zone found lateral to the traversing nerve root and just medial to the exiting involved nerve root coupled with a more shallow approach to the involved disc as compared to the 
angle used when performing discograms (Alo), endoscopic discectomy and neural decompression was performed. Integral to the surgical procedure was the subsequent randomization of allograft tissue implantation to adjuvantly address inflammation and promote healing/functional recovery.

Intraoperative discogram was performed. Endoscopic visualization (2.5-5 mm fixed magnifying camera) of disc herniation was noted. Disc cannula system utilized was comprised of an array (3.3-6.9 $\mathrm{mm})$ of tissue separators governed and protected over progressive soft tissue dilators. Intradiscal decompression was performed utilizing an array of pituitary graspers $(2.5-4 \mathrm{~mm})$ coupled with energy based electrocautery agents (bipolar, high frequency radio frequency, low frequency radio frequency, quantum molecular, laser.

\section{Considerations}

Treatment selection remains paramount when considering treatment options. In this study, an intact posterior longitudinal ligament coupled with $8 \mathrm{~mm}$ AP diameter or greater coupled with radicular symptomology was utilized. The methods of lumbar microdiscectomy coupled with physician technique are equally important aspects. Although, a consistent physical therapy regimen was included in the treatment population, nonetheless, rehabilitative techniques may be a confounding factor. A Body Mass Index of less than 25 was used in the treatment population.

Additionally, In prior studies, the use of bone marrow aspirate has additional concerns including age-related decline and thus population number and efficacy (Ajiboye RM). However, favorable fusion rates were nonetheless seen (Ajiboye RM). Route of allograft tissue/ mesenchymal stem cells obtainment is thus a consideration. Furthermore, prior studies have revealed efficacy with use of Platelet Rich Plasma in disc pathology. Similarly, the concomitant use of adipocyte mesenchymal stem cells in combination with PRP has been examined with functional benefit as well (Panganiban).

Dosing considerations remain in play. In this study, $2 \mathrm{cc}$ of human allograft tissue was utilized intradiscally. Indeed, prior literature, has suggested a nonlinear benefit. Minimal numbers of cells may be adequate. In effect, increased dose amounts does not equate to improved and commensurate benefit in turn. Furtherance of study delineating alternative treatment models inclusive of dosing ranges are needed.

\section{Summary}

There is a high morphological variability in disc pathology in symptomatic patients. Prior studies have quoted as much as 20 percent under 40 years of age have a diagnosed disc herniation and 50 percent over 50 years of age having a diagnosed disc herniation, facet degenerative joint disease and concomitant neuroforaminal stenosis (Weissel). Over 60 years of age, at least 35 percent have multilevel involvement concomitantly with 90-93 percent involving L4-5 and/or L5-S1 and are central in the spinal canal with the onset of radicular symptoms with concomitant functional deficits, however, early intervention addressing the inflammatory cascade and promotion of healing are paramount [1-7].

The concept of a targeted multimodal analgesic regimen continues to be a fixture in the management of pain. Incorporating rehabilitative, medicinal, interventional/surgical means to reduce pain and more importantly restore function al independence remains the hallmark for current and future treatment in this treatment field.

Intervertebral disc pathology contributes to the "InflammatoryImmune" model oftentimes accompanied with hallmark nerve inflammation and neuropathic pain. With disc herniation, early TNFalpha release secondary to inflammation leads to ectopic firing of ion channels at the dorsal root ganglion. This, coupled with resultant immunomodulation and peripheral sensitization, forms the basis for eventual central sensitization (substance $\mathrm{P} /$ glutamate) if untreated at the dorsal horn of the spinal cord. Indeed, neurodegenerative processes as a whole have had a postulated link to neural stem cell repair via immunomodulatory aspects (C3a link during ischemic stroke and neurogenesis)(Stokowska A). bFGF has been postulated, similarly, via ERK/MAPK signal upregulation secondary to inflammation in neuronal injury (Traumatic Brain Injury), to promote neuronal stem cell migration and repair (Duan $\mathrm{H}$ ). T-cell mediated inflammation adressment has been studied in neurodegenerative disease (Multiple Sclerosis)(Benvenuto F). Thus, Recovery/treatment strategies in Spine have been sought addressing this neuroimmunomodulatory component to the inflammatory-immune model. As an extension, Minimally-invasive treatment options to assist in functional restoration and reduction of pain, when appropriate, have thus become a growing mainstay of consideration and inclusion. Moreover, more invasive treatment options including fusion are oftentimes associated with complications of pseudoarthrosis and adjacent segment degeneration again due to inflammation promotion enhancing the degenerative cascade (Zhang ZM). The overall reoperation rate as well as the adressment of the newly-involved segment levels, were found to be increased (Zhang ZM). Thus, a diverse array of minimally-invasive treatment options are being studied and utilized. Favorable if not comparable results when juxtaposed with open discectomy have frequently been seen (Manchikanti). With functional restoration of basic and advanced activities of daily living becoming one of the main gold standards in treatment goals and measuring of treatment efficacy, amplification of healing mechanisms coupled with implementation of rehabilitation principles in keeping with a multimodal approach is fast becoming the norm.

To adjuvantly address inflammation and to assist in the promotion of healing, the implementation of allograft tissue (and other regenerative medicine considerations) as part of a treatment strategy continues to grow in favor as a result. In keeping then, pervasive thought identifying Cartilage/Connective tissue loss integral at the core of the degenerative cascade may be helped from a regenerative/ rejuvenatory capacity with its inclusion then. Allograft/Stem cell treatment has been prior studied in Anterior cervical discectomy and cervical/lumbar spine fusion (Hansraj KK). Stem cell augmentation was seen favorably when compared to the gold standard to bone graft (iliac crest)(Hansraj KK). Similarly, it was intimated that Mesenchymal stem cell implantation showed improvement with discogenic pain (Hansraj). With increased structural stabilization, repair may be enhanced allowing for inclusion of the third aspect of the multimodal treatment regimen. Physical rehabilitation, too, in keeping with the targeted multimodal analgesic approach, may play a significant role in stem cell repair via inflammatory-immune means. Increased VEGF levels with increased aerobic activity (treadmill) have been found. It is postulated that a neural stem cell repair link may be involved (Zhao) [8-10].

In summary, A difference in statistical significance ( $p$ less than 0.05) was seen at six months with regards to SF-36 (bodily pain, Role- 
Citation: Rudy P (2017) Endoscopic Discectomy with Amniotic Allograft Tissue Implantation for Treatment of Lumbar Disc Herniation. J Pharmacogenomics Pharmacoproteomics 8: 174. doi:10.4172/2153-0645.1000174

Page 3 of 3

Physical) measurement when compared to those positive responders who did not receive allograft tissue implantation. The relevance and implied benefit of minimally-invasive techniques as part of a targeted multimodal analgesic regimen addressing the inflammatory-immune model may extend then not only with absolute pain levels but also with overall functional independence with inclusion of regenerative medicine considerations such as allograft tissue implantation. Indeed, sustained functional restoration/recovery may be seen. Neural control and influence on the immune system continues to be studied. Neuromodulation (Vagus nerve), as an example, has been studied with implied influence on the locus ceruleus and thus Norepinephrine levels with downstream effects on GABA and hence the descending pain inhibitory pathways which have mitochondrial immune (macrophage $\mathrm{T}$ cell/Interleukin) considerations. The implied need for a targeted multimodal analgesic approach with the inclusion of adjuvant means/ modalities is thus also suggested. Further longitudinal study is supported.

\section{References}

1. Ajiboye R, Zoller S, Ashana A, Sharma A, Sheppard W, et al. (2017) Regression of Disc-Osteophyte Complexes Following Laminoplasty Versus Laminectomy with Fusion for Cervical Spondylotic Myelopathy International Journal of Spine Surgery. Int J Spine Surg 11: 129-137.
2. Kenneth A (2005) Percutaneous Lumbar discectomy. Pain Practice.

3. Benvenuto F, Voci A, Carminati E, Gualandi F, Mancardi G, et al. (2015) Human mesenchymal stem cells target adhesion molecules and receptors involved in T cell extravasation. Stem Cell Res Ther. Springer Nature 6: 1.

4. Ye X, Ju S, Duan H, Yao Y, Wu J, et al. (2017) Immune checkpoint molecule PD-1 acts as a novel biomarker for the pathological process of gestational diabetes mellitus. Biomark Med. Future Medicine 11: 741-749.

5. Errico TJ, Fardon DF, Lowell TD, Vaccaro A (2003) Open discectomy as treatment for herniated nucleus pulposus of the lumbar spine. Spine J. Elsevier BV 3: 45-49.

6. Hansraj KK (2016) Stem cells in Spine Surgery. Surgery Technol Int.

7. Manchikanti L, Cash, McManus, Pampati, Benyamin (2012) Fluoroscopic lumbar interlaminar epidural injections in managing chronic lumbar axial or discogenic pain. J Pain Res. Dove Medical Press 301.

8. Beattie PF, Brooks WM, Rothstein JM, Sibbitt WL, Robergs RA, et al. ( 1994) Effect of Lordosis on the Position of the Nucleus Pulposus in Supine Subjects. Spine Ovid Technologies 19: 2096-2102.

9. Feng Y, Chen L, Gu Y, Zhang Z-M, Yang H-L, et al. (2015) Restoration of the spinopelvic sagittal balance in isthmic spondylolisthesis: posterior lumbar interbody fusion may be better than poster lateral fusion. Spine J . Elsevier BV 15:1527-1535.

10. Cheng X, Zhang G, Zhang L, Hu Y, Zhang K, et al. (2017) Mesenchymal stem cells deliver exogenous miR-21 via exosomes to inhibit nucleus pulposus cell apoptosis and reduce intervertebral disc degeneration. J Cell Mol Med. Wiley-Blackwell. 